\title{
Bipolaron recombination in conjugated polymers
}

Zhen Sun and Sven Stafström

\section{Linköping University Post Print}

N.B.: When citing this work, cite the original article.

Original Publication:

Zhen Sun and Sven Stafström, Bipolaron recombination in conjugated polymers, 2011, Journal of Chemical Physics, (135), 7, 074902.

http://dx.doi.org/10.1063/1.3624730

Copyright: American Institute of Physics (AIP) http://www.aip.org/

Postprint available at: Linköping University Electronic Press

http://urn.kb.se/resolve?urn=urn:nbn:se:liu:diva-70527 


\title{
Bipolaron recombination in conjugated polymers
}

\author{
Zhen Sun a) and Sven Stafström \\ Department of Physics, Chemistry and Biology, Linköping University, SE-58183 Linköping, Sweden
}

(Received 19 March 2011; accepted 22 July 2011; published online 19 August 2011)

\begin{abstract}
By using the Su-Schrieffer-Heeger model modified to include electron-electron interactions, the Brazovskii-Kirova symmetry breaking term and an external electric field, we investigate the scattering process between a negative and a positive bipolaron in a system composed of two coupled polymer chains. Our results show that the Coulomb interactions do not favor the bipolaron recombination. In the region of weak Coulomb interactions, the two bipolarons recombine into a localized excited state, while in the region of strong Coulomb interactions they can not recombine. Our calculations show that there are mainly four channels for the bipolaron recombination reaction: (1) forming a biexciton, (2) forming an excited negative polaron and a free hole, (3) forming an excited positive polaron and a free electron, (4) forming an exciton, a free electron, and a free hole. The yields for the four channels are also calculated. (C) 2011 American Institute of Physics. [doi:10.1063/1.3624730]
\end{abstract}

\section{INTRODUCTION}

Bipolarons are important charge carriers in conjugated polymers. They were hypothesized to exist in conjugated polymers almost 30 years ago and were extensively studied over the years. ${ }^{1}$ Several experiments have demonstrated the existence of bipolarons, especially in doped polymers. ${ }^{2-5}$ Some of the early studies concentrated on the stability of bipolaron. ${ }^{6-10}$ It is now clear that both the electron-phonon coupling and the electron-electron interaction play an important role in forming bipolarons: at low concentration of carriers, carriers are more likely in the form of polarons, while at high concentration of carriers they tend to favor bipolarons. ${ }^{6}$ Bipolarons may also coexist with polarons in conjugated polymers. Total energy calculations indicate that two extra electrons may go either into two independent polarons or into a bipolaron. ${ }^{11}$ There is a subtle balance between the two situations. Optical and magnetic data of some polymers showed signals involving polarons and bipolarons, ${ }^{12-14}$ which testify the coexistence of polarons and bipolarons in conjugated polymers.

In recent years, there has been a great interest in the use of conjugated polymers in light-emitting diodes (LEDs). ${ }^{15}$ To improve the efficiency of LED, much attention is focused to the polaron recombination process. Polarons are injected from anode and cathode of the LED and recombine to form singlet or triplet excitons. Triplet excitons are non-emissive, while singlet excitons are emissive which gives rise to electroluminescence of the LED. According to spin statistics, the singletto-triplet formation ratio will be $1: 3$. Hence, the electroluminescence efficiency is limited to $25 \%$. However, a number of reports have indicated that the electroluminescence efficiency in LEDs ranges between $22 \%$ and $83 \%$, which greatly exceeds the theoretical limitation predicted by spin statistics. ${ }^{16-20}$ The reason for that is still under investigation.

\footnotetext{
a)Electronic mail: zhesu@ifm.liu.se.
}

In organic LEDs, in which the carrier concentration is relatively low, polaron recombination is believed to be the normal electroluminescence channel. However, with the coexistence of polarons and bipolarons in mind, it is straightforward to conjecture a similar process where a negative bipolaron and a positive bipolaron recombine into a biexciton. Theoretical and experimental evidences for stable biexcitons in conjugated polymers have been reported in the literatures, ${ }^{21-24}$ which indicate that they might also exist as a result of bipolaron recombination.

Using a combined version of the Su-Schrieffer-Heeger (SSH) model $^{25}$ and the extended Hubbard model, we simulated the scattering process between a negative and a positive bipolaron in two coupled polymer chains in the presence of an external electric field. The simulations were performed using a nonadiabatic molecular dynamics method which was introduced by Ono and Terai ${ }^{26}$ We have applied this method to the studies of scattering processes between polaron and exciton, ${ }^{27}$ polaron and bipolaron, ${ }^{28}$ bipolaron and exciton. ${ }^{29}$ The aim of this paper is to give a microscopic picture of the bipolaron recombination process as well as to testify the products of the bipolaron recombination.

\section{MODEL AND METHOD}

The SSH-type Hamiltonian modified to include electronelectron interactions and an external electric field is given by

$$
H=H_{\text {elec }}+H_{\text {latt }}=H_{e l}+H_{e e}+H_{E}+H_{\text {latt }} .
$$

In Eq. (1), $H_{e l}$ is the SSH Hamiltonian,

$$
H_{e l}=-\sum_{n, n^{\prime}, s} t_{n, n^{\prime}} c_{n, s}^{\dagger} c_{n^{\prime}, s}
$$

The operator $c_{n, s}^{\dagger}\left(c_{n, s}\right)$ creates (annihilates) a $\pi$ electron with spin $s$ at the $n$th site. $t_{n, n^{\prime}}$ is the hopping integral between site 
$n$ and $n^{\prime}$ :

$$
t_{n, n^{\prime}}= \begin{cases}t_{0}-\alpha\left(u_{n}-u_{n^{\prime}}\right)+(-1)^{n} t_{e}, & \text { intrachain hopping with } \mathrm{n}^{\prime}=\mathrm{n} \pm 1 \\ t_{\perp} \text { or } t_{d}, & \text { interchain hopping }\end{cases}
$$

where $t_{0}$ is the hopping integral of $\pi$ electrons for zero lattice displacements, $\alpha$ the electron-phonon coupling constant, $u_{n}$ the lattice displacement of the $n$th site from its equidistant position, and $t_{e}$ is introduced to lift the ground-state degeneracy for non-degenerate polymers. $t_{\perp}$ is the orthogonal hopping integral, i.e., the hopping between a site on one chain and a nearest neighbor site on an adjacent chain, and $t_{d}$ the diagonal hopping integral, i.e., the hopping between next nearest neighbor site on adjacent chains.

The term $H_{e e}$ in Eq. (1) expresses the electron-electron interactions limited to an extended-Hubbard-type expression including on-site and nearest-neighbor Coulomb interactions,

$$
\begin{aligned}
H_{e e}= & U_{0} \sum_{n, s}\left(c_{n, s}^{\dagger} c_{n, s}-\frac{1}{2}\right)\left(c_{n,-s}^{\dagger} c_{n,-s}-\frac{1}{2}\right) \\
& +U_{n, n^{\prime}} \sum_{n, n^{\prime} s, s^{\prime}}\left(c_{n, s}^{\dagger} c_{n, s}-\frac{1}{2}\right)\left(c_{n^{\prime}, s^{\prime}}^{\dagger} c_{n^{\prime}, s^{\prime}}-\frac{1}{2}\right),
\end{aligned}
$$

where $U_{0}$ and $U_{n, n^{\prime}}$ are the on-site and nearest-neighbor Coulomb interaction strengths, respectively. $U_{n, n^{\prime}}$ is expressed as

$$
\begin{aligned}
& U_{n, n^{\prime}} \\
& = \begin{cases}V, & \text { intrachain Coulomb interaction with } \mathrm{n}^{\prime}=\mathrm{n} \pm 1, \\
V_{\perp}, & \text { interchain nearest-neighbor Coulomb interaction. }\end{cases}
\end{aligned}
$$

In this paper, these extended-Hubbard-type interactions are treated within the Hartree-Fock approximation.

The electric field is included in the Hamiltonian as a scalar potential, which gives the following contribution to the Hamiltonian:

$$
H_{E}=|e| E \sum_{n, s}\left(n a+u_{n}\right)\left(c_{n, s}^{\dagger} c_{n, s}-\frac{1}{2}\right)
$$

where $E$ is the external electric field, $e$ the absolute value of electronic charge and $a$ the lattice constant.

The lattice energy is described by

$$
H_{\text {latt }}=\frac{1}{2} K \sum_{n}\left(u_{n+1}-u_{n}\right)^{2}+\frac{1}{2} M \sum_{n} \dot{u}_{n}^{2}
$$

where $K$ is the elastic constant of a $\sigma$ bond and $M$ the mass of a $\mathrm{CH}$ group.

The model parameters we use in this work are those generally chosen for polyacetylene, ${ }^{1} t_{0}=2.5 \mathrm{eV}, \alpha=4.1 \mathrm{eV} / \AA$, $t_{e}=0.05 \mathrm{eV}, K=21.0 \mathrm{eV} / \AA^{2}, M=1349.14 \mathrm{eVfs}^{2} / \AA^{2}, a$ $=1.22 \AA, t_{\perp}=0.1 \mathrm{eV}$, and $t_{d}=0.05 \mathrm{eV}$.

The on-site Coulomb interaction is expressed as $U_{0}$ $=f t_{0}$, where in this work calculations are performed for $f$ $=0$ to 1.5 . Larger values of $f$ lead to destabilization of the bipolarons (see Sec. III). The value for the intrachain nearestneighbor Coulomb interaction $V$ is set to $U_{0} / 3$, that is $f t_{0} / 3$, and the value for the interchain nearest-neighbor Coulomb interaction $V_{\perp}$ is set to $f t_{\perp} / 3$. In all the simulations, the external electric field strength is set to $5 \times 10^{4} \mathrm{~V} / \mathrm{cm}$.

The time-dependent Schrödinger equations for oneparticle wavefunctions are

$$
i \hbar \frac{\partial}{\partial t} \psi_{k}(n, t)=H_{e l e c} \psi_{k}(n, t),
$$

where $k$ is the quantum number that specifies an electronic state. The equation of motions for the lattice sites are

$$
\begin{aligned}
M \ddot{u}_{n}= & F_{n}(t)=-K\left[u_{n+1}(t)-u_{n-1}(t)-2 u_{n}(t)\right] \\
& +\alpha[\rho(n, n+1, t)-\rho(n-1, n, t)] \\
& +|e| E[\rho(n, t)-1]-\lambda M \dot{u}_{n},
\end{aligned}
$$

where $F_{n}(t)$ represents the force that the $n$th site endured. The damping of the motion describes the dissipation of energy gained from the electric field. The damping constant $\lambda$ is set to $0.001 \mathrm{fs}^{-1}$ in our calculations. ${ }^{30}$ The charge density $\rho\left(n, n^{\prime}, t\right)$ is expressed as

$$
\rho\left(n, n^{\prime}, t\right)=\sum_{k}^{\prime} \psi_{k}^{*}(n, t) f_{k} \psi_{k}\left(n^{\prime}, t\right)
$$

where $f_{k}$ is the time-independent distribution function of 0,1 , or 2 depending on initial state occupation.

The above set of equations are numerically solved by discretizing the time with an interval $\Delta t$ which is chosen to be sufficiently small so that the change of the electronic Hamiltonian during that interval may be negligible. For all the results presented below, we choose a time step of $\Delta t=0.005 \mathrm{fs}$.

By introducing instantaneous eigenstates, the solutions of the time-dependent Schrödinger equations can be put in the form $^{26}$

$$
\psi_{k}\left(n, t_{j+1}\right)=\sum_{l}\left[\sum_{m} \phi_{l}^{*}(m) \psi_{k}\left(m, t_{j}\right)\right] e^{-i\left(\epsilon_{l} \Delta t / \hbar\right)} \phi_{l}(n)
$$

where $\left\{\phi_{l}(n)\right\}$ and $\left\{\epsilon_{l}\right\}$ are the eigenfunctions and eigenvalues of the Hamiltonian $H_{\text {elec }}$ at a given time $t_{j}$. The lattice 
equations are written as

$$
\begin{gathered}
u_{n}\left(t_{j+1}\right)=u_{n}\left(t_{j}\right)+\dot{u}_{n}\left(t_{j}\right) \Delta t, \\
\dot{u}_{n}\left(t_{j+1}\right)=\dot{u}_{n}\left(t_{j}\right)+\frac{F_{n}\left(t_{j}\right)}{M} \Delta t .
\end{gathered}
$$

Hence, the electronic wave functions and the lattice displacements at the $(j+1)$ th time step are obtained from the $j$ th time step.

At a given time $t_{j}$, the wave functions $\left\{\psi_{k}\left(n, t_{j}\right)\right\}$ can be expressed as a series expansion of the eigenfunctions $\left\{\phi_{l}\right\}$ :

$$
\psi_{k}\left(n, t_{j}\right)=\sum_{l=1}^{N} C_{l, k}\left(t_{j}\right) \phi_{l},
$$

where $C_{l, k}$ are the expansion coefficients. The occupation number for energy level $\varepsilon_{l}$ is

$$
n_{l}\left(t_{j}\right)=\sum_{k}^{\prime} f_{k}\left|C_{l, k}\left(t_{j}\right)\right|^{2} .
$$

$n_{l}\left(t_{j}\right)$ contains information concerning the redistribution of electrons among the energy levels.

In all simulations, we use the staggered order parameter $r_{n}(t)$ and the mean charge density $\bar{\rho}_{n}(t)$ to analyze the lattice and charge density evolution,

$$
\begin{aligned}
r_{n}(t)= & \frac{(-1)^{n}}{4}\left[u_{n-1}(t)+u_{n+1}(t)-2 u_{n}(t)\right], \\
\bar{\rho}_{n}(t)= & \frac{1}{4}[\rho(n-1, n-1, t)+2 \rho(n, n, t) \\
& +\rho(n+1, n+1, t)] .
\end{aligned}
$$

To simulate the bipolaron recombination process, we must first obtain two opposite charged bipolarons on two coupled polymer chains, respectively. In our simulation, each chain has 200 sites. The sites in chain 1 are labeled 1-200, while the sites in chain 2 are labeled 201-400. As shown in Fig. 1(a), the two chains are placed beside each other and overlap by 100 sites: the 201 th to 300 th sites are coupled with the 101th to 200th sites, respectively. The starting geometry is obtained by minimizing the total energy of the system with fixed occupation numbers as described in Fig. 1(b) without the presence of the electric field. The negative bipolaron is located at the site 50 (in chain 1) while the positive bipolaron is at site 350 (in chain 2). With the electric field being smoothly turned on, the two opposite charged bipolarons begin to move towards each other, then collide.

\section{RESULTS AND DISCUSSIONS}

In Fig. 2, we present the temporal evolution of staggered order parameter $r_{n}(t)$ (left panel) and mean charge density $\bar{\rho}_{n}(t)$ (right panel) for the bipolaron scattering process with different on-site Coulomb interactions. Panels a1 and a2 correspond to $U_{0}=0.2 t_{0}(0.5 \mathrm{eV})$, panels b1 and b2 $U_{0}=0.3 t_{0}$ $(0.75 \mathrm{eV})$ and panels $\mathrm{c} 1$ and $\mathrm{c} 2 U_{0}=0.4 t_{0}(1.0 \mathrm{eV})$. We can see that the bipolaron scattering processes are quite different while the on-site Coulomb interaction $U_{0}$ increases. In the

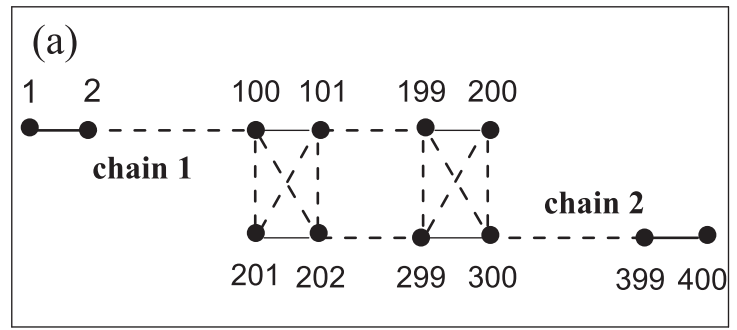

(b)

Unoccupied conduction band

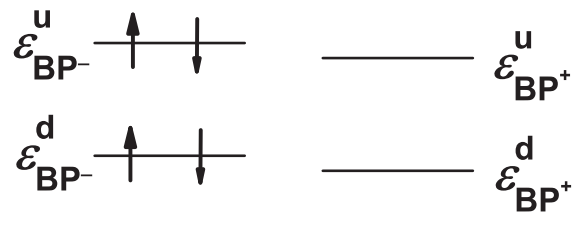

\section{Double occupied valence band}

FIG. 1. Schematic diagram of (a) two coupled chains and (b) the intra-gap energy levels and their occupations of the system containing a negative and a positive bipolaron. There are four intra-gap levels: the left two $\varepsilon_{\mathrm{BP}^{-}}^{\mathrm{u}}$ and $\varepsilon_{\mathrm{BP}^{-}}^{\mathrm{d}}$ come from the negative bipolaron and each of them is doubly occupied; the right two $\varepsilon_{\mathrm{BP}^{+}}^{\mathrm{u}}$ and $\varepsilon_{\mathrm{BP}^{+}}^{\mathrm{d}}$ come from the positive bipolaron and both of them are empty.

case of $U_{0}=0.2 t_{0}$, the two bipolarons begin to interact at about 500 fs. Then, they quickly recombine and form a localized excited state on chain 1 . As a result of this recombination, chain 2 ends up on the potential energy surface of the neutral ground state (see panel a2) and slowly reaches equilibrium as a result of the energy dissipation from the system due to the damping. From panel a2, we see that both chains become essentially neutral after recombination apart from small intra chain charge density fluctuations associated with the localized excitation on chain 1 .

In Fig 2, panels b1 and b2, the on-site Coulomb interaction $U_{0}$ is increased to $0.3 t_{0}$. The two bipolarons repel each other when they first interact at about 500 fs. Under the influence of the external electric field, they move towards each other a second time and interact again at about $900 \mathrm{fs}$. This time they recombine in a way very similar to the process described above. If the on-site Coulomb interaction $U_{0}$ increases to $0.4 t_{0}$, we see that the two biplarons never recombine, as shown in Fig. 2 panels $\mathrm{c} 1$ and c2. As a result of the Coulomb interaction, the two bipolarons are attracted towards each other and loose their initial kinetic energy after repeated collisions.

If we continue increasing the on-site Coulomb interaction $U_{0}$ above $0.4 t_{0}$ we observe no significant change in the qualitative behavior of the scattering process, i.e., for this strength of the Coulomb interactions, the two bipolarons cannot recombine. Thus, we can conclude that Coulomb interactions are unfavorable for bipolaron recombination. We also observe from our simulations that for $U_{0}$ values above $1.5 t_{0}$, the bipolarons themselves become unstable. Since the existence of 
(a1)

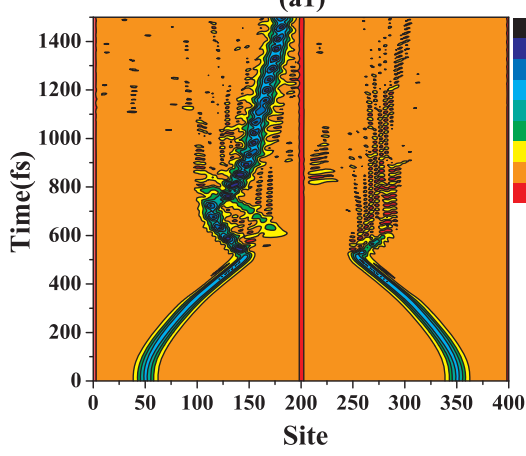

(b1)

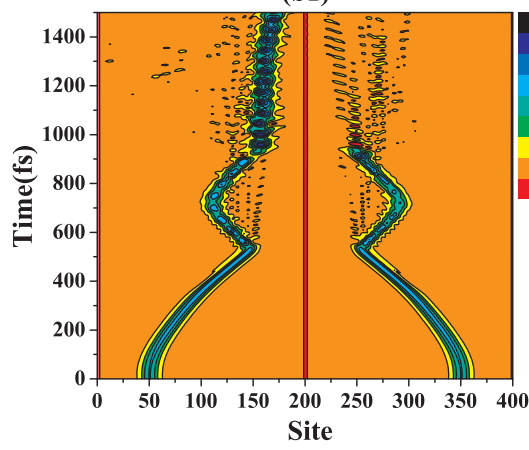

(c1)

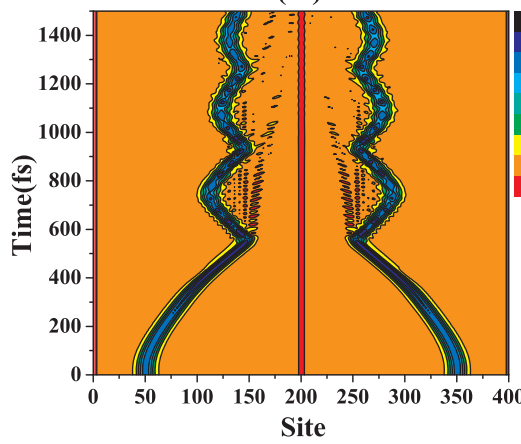

(a2)
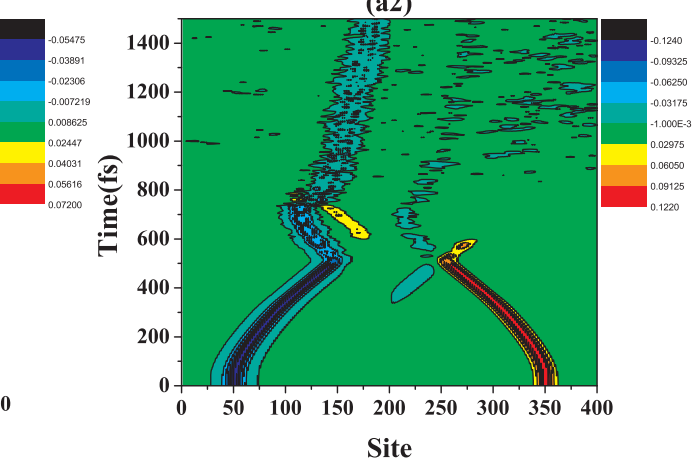

(b2)
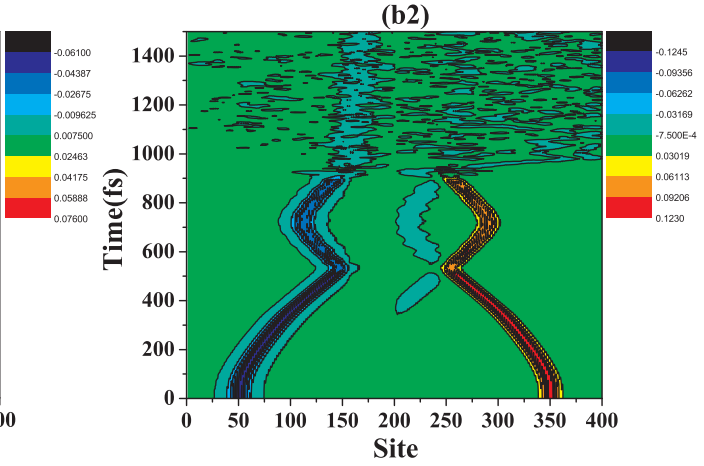

(c2)

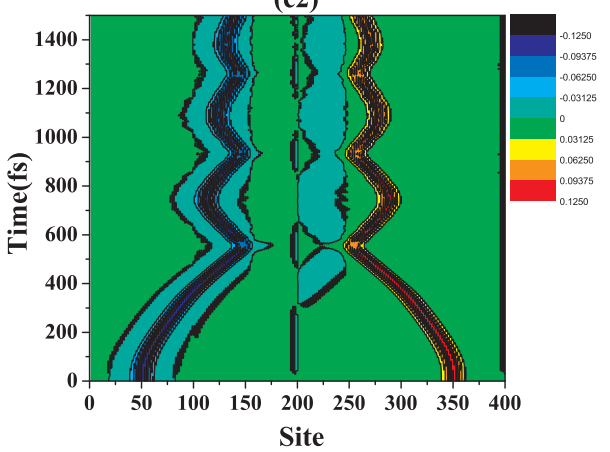

FIG. 2. Time dependence of $r_{n}$ (left panel) and $\bar{\rho}_{n}$ (right panel) for the bipolaron recombination process with different $U_{0}$ values: $U_{0}=0.2 t_{0}$ (top panel), $U_{0}=0.3 t_{0}$ (middle panel), and $U_{0}=0.4 t_{0}$ (bottom panel).

bipolarons is well documented in the literature, we believe that such large values of $U_{0}$ lie outside the physically relevant regime.

In order to further investigate the influence of the Coulomb potential we also performed calculations using a long range interaction "tail" added to the originally proposed on-site (U) and nearest neighbor (V) potential. The results from these calculations are very similar to those presented above showing that the short range terms to a large extent determine the dynamics of the bipolaron recombination process.

To understand the behavior of the two bipolarons during the scattering processes and address the properties of the localized excitation formed after recombination, it is useful to view the changes of the electronic structure of the system during and after the recombination process. For the cases depicted in Fig. 2, we show in Fig. 3 the time evolutions of the intra-gap levels and their occupation numbers during the bipolaron scattering process. As seen from the left panel, at the beginning there are four intra-gap levels caused by the two bipolarons. According to their wave functions, we know that the red and blue lines correspond to the negative bipolaron levels $\varepsilon_{\mathrm{BP}^{-}}^{\mathrm{u}}$ and $\varepsilon_{\mathrm{BP}^{-}}^{\mathrm{d}}$, respectively, while the green and dark green levels correspond to the positive bipolaron levels $\varepsilon_{\mathrm{BP}^{+}}^{\mathrm{u}}$ and $\varepsilon_{\mathrm{BP}^{+}}^{\mathrm{d}}$, respectively. From the right panel of Fig. 3, we see that at the beginning $\varepsilon_{\mathrm{BP}^{-}}^{\mathrm{u}}$ and $\varepsilon_{\mathrm{BP}^{-}}^{\mathrm{d}}$ are doubly occupied, while $\varepsilon_{\mathrm{BP}^{+}}^{\mathrm{u}}$ and $\varepsilon_{\mathrm{BP}^{+}}^{\mathrm{d}}$ are empty. For clarity, we have used the same color coding but with dashed (blue), dotted (green), and dashed-dotted (red) lines in the right panel. With the external electric field smoothly applied during the first 50 fs, $\varepsilon_{\mathrm{BP}^{-}}^{\mathrm{u}}$ and $\varepsilon_{\mathrm{BP}^{-}}^{\mathrm{d}}$ move upward in energy, while $\varepsilon_{\mathrm{BP}^{+}}^{\mathrm{u}}$ and $\varepsilon_{\mathrm{BP}^{+}}^{\mathrm{d}}$ move downward. When the field strength has reached a constant value, the dynamics is completely determined by intrinsic effects.

Let us focus on Fig. 3 panels b1 and b2 $U_{0}=0.3 t_{0}$. At about 500 fs, i.e., the instant of the first encounter of the two bipolarons, the four intra-gap levels begin to oscillate due to the electron-phonon coupling and the interaction between the bipolarons. At the same time, their occupation numbers change slightly corresponding to a small charge transfer 
(a1)

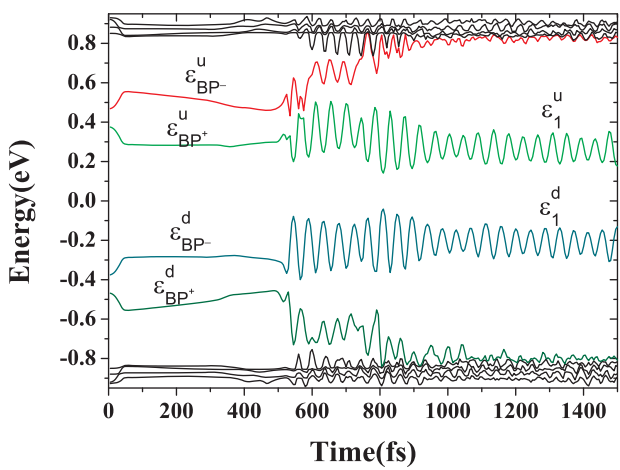

(b1)

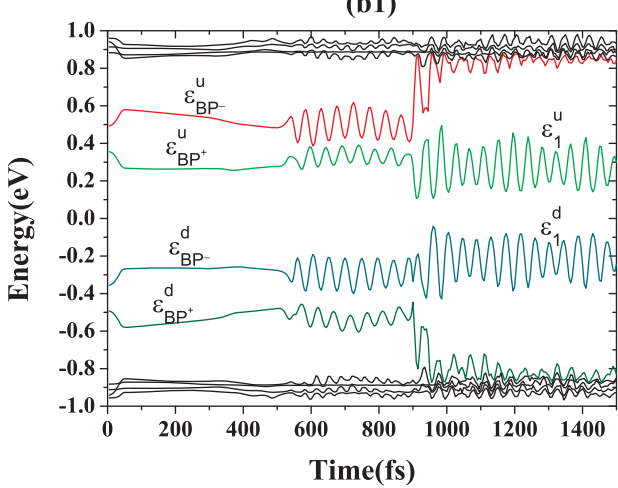

(c1)

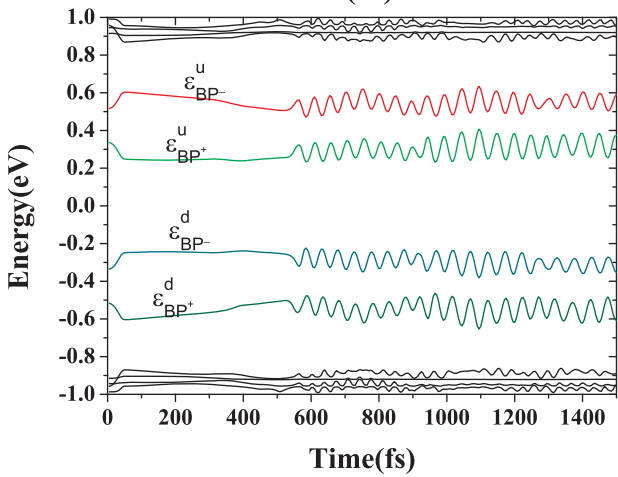

(a2)

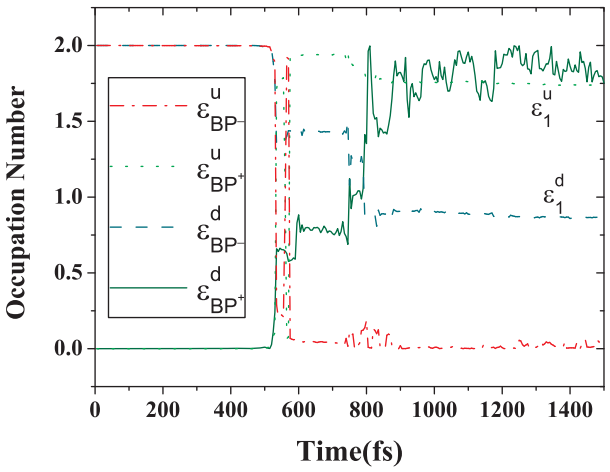

(b2)

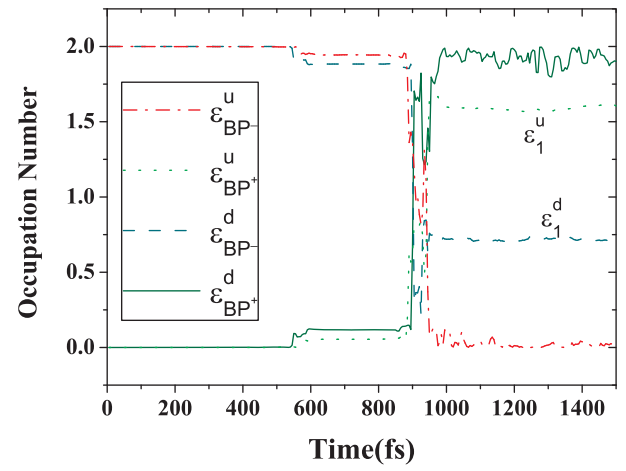

(c2)

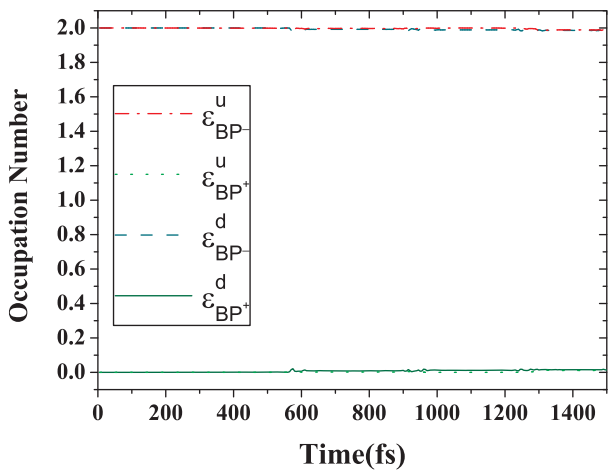

FIG. 3. Time evolutions of the intra-gap levels (left panel) and their occupation numbers (right panel) during the bipolaron recombination process for different $U_{0}$ values: $U_{0}=0.2 t_{0}$ (top panel), $U_{0}=0.3 t_{0}$ (middle panel), and $U_{0}=0.4 t_{0}$ (bottom panel).

between the two chains. At about 900 fs, i.e., the instant of the second encounter of the two bipolarons, $\varepsilon_{\mathrm{BP}^{-}}^{\mathrm{u}}$ and $\varepsilon_{\mathrm{BP}^{+}}^{\mathrm{d}}$ move to the conduction band and the valence band, respectively, whereas $\varepsilon_{\mathrm{BP}^{+}}^{\mathrm{u}}$ and $\varepsilon_{\mathrm{BP}^{-}}^{\mathrm{d}}$ move towards the midgap region. Accordingly, the occupation numbers of these intra-gap levels also change dramatically: the occupation numbers of $\varepsilon_{\mathrm{BP}^{-}}^{\mathrm{u}}$ and $\varepsilon_{\mathrm{BP}^{-}}^{\mathrm{d}}$ reduce to about zero and 0.6 , respectively, and the occupation numbers of $\varepsilon_{\mathrm{BP}^{+}}^{\mathrm{u}}$ and $\varepsilon_{\mathrm{BP}^{+}}^{\mathrm{d}}$ increase to about 1.7 and 2.0, respectively. After $900 \mathrm{fs}$, since the two bipolarons have recombined into a localized excited state, $\varepsilon_{\mathrm{BP}^{+}}^{\mathrm{u}}$ and $\varepsilon_{\mathrm{BP}^{-}}^{\mathrm{d}}$ no longer correspond to the bipolarons. To avoid confusion, we rename these states as $\varepsilon_{1}^{\mathrm{u}}$ and $\varepsilon_{1}^{\mathrm{d}}$, respectively, see Fig. 3.

A third change that occurs during the bipolaron recombination is a relocation of the eigenfunctions associated with the bipolarons. After $900 \mathrm{fs}$, the eigenfunctions of $\varepsilon_{\mathrm{BP}^{-}}^{\mathrm{u}}$ and $\varepsilon_{\mathrm{BP}^{+}}^{\mathrm{d}}$ delocalize on the two chains whereas the eigenfunction of $\varepsilon_{\mathrm{BP}^{+}}^{\mathrm{u}}\left(\varepsilon_{1}^{\mathrm{u}}\right)$ relocates from chain 2 to chain 1 . The eigenfunction of $\varepsilon_{\mathrm{BP}^{-}}^{\mathrm{d}}\left(\varepsilon_{1}^{\mathrm{d}}\right)$, however, remains localized on chain 1 during the entire recombination process.

To see explicitly the electron transfer among energy levels, we show the mean occupation numbers of the levels around the gap after the bipolaron recombination in Table I. We can see that a large number of levels in conduction and valence band, beside the intra-gap levels, are involved in the bipolaron recombination. These results show that there are certain probabilities of forming free electrons and holes after the bipolaron recombination.

From Fig. 3 panel $\mathrm{c} 1$ and $\mathrm{c} 2$, we see that the four intra-gap levels all oscillate after the two bipolarons collide, however, their occupation numbers hardly change. This is in accordance with the results that the two bipolarons never recombine in the case of strong Coulomb interactions. To 
TABLE I. The mean occupation numbers of the levels around the gap (from level 189 to 212), which are calculated after the bipolaron recombination (from $1000 \mathrm{fs}$ to $1500 \mathrm{fs}), U_{0}=0.3 t_{0}$.

\begin{tabular}{cccccccccccc}
\hline \hline 200 & 199 & 198 & 197 & 196 & 195 & 194 & 193 & 192 & 191 & 190 & 189 \\
\hline 0.716 & 1.925 & 1.926 & 1.945 & 1.938 & 1.954 & 1.974 & 1.962 & 1.974 & 1.977 & 1.969 & 1.970 \\
201 & 202 & 203 & 204 & 205 & 206 & 207 & 208 & 209 & 210 & 211 & 212 \\
1.589 & 0.0191 & 0.0239 & 0.0190 & 0.0165 & 0.0215 & 0.0192 & 0.0266 & 0.0270 & 0.0194 & 0.0139 & 0.0107 \\
\hline \hline
\end{tabular}

explain why the Coulomb interactions do not favor bipolaron recombination, we see that the energy shift between $\varepsilon_{\mathrm{BP}^{-}}^{\mathrm{u}}$ and $\varepsilon_{\mathrm{BP}^{+}}^{\mathrm{u}}\left(\right.$ or $\varepsilon_{\mathrm{BP}^{-}}^{\mathrm{d}}$ and $\left.\varepsilon_{\mathrm{BP}^{+}}^{\mathrm{d}}\right)$ is increasing with the on-site Coulomb interaction $U_{0}$. If the energy shift becomes too large (equal or greater than $0.4 t_{0}$ ), the two levels $\varepsilon_{\mathrm{BP}^{-}}^{\mathrm{u}}$ and $\varepsilon_{\mathrm{BP}^{+}}^{\mathrm{u}}$ (or $\varepsilon_{\mathrm{BP}^{-}}^{\mathrm{d}}$ and $\varepsilon_{\mathrm{BP}^{+}}^{\mathrm{d}}$ ) will not be able to interact. In other word, electron transition from $\varepsilon_{\mathrm{BP}^{-}}^{\mathrm{u}}$ to $\varepsilon_{\mathrm{BP}^{+}}^{\mathrm{u}}$ (or from $\varepsilon_{\mathrm{BP}^{-}}^{\mathrm{d}}$ to $\varepsilon_{\mathrm{BP}^{+}}^{\mathrm{d}}$ ) becomes impossible and the bipolarons can not recombine.

The above results show clearly that the bipolaron recombination is associated with inter-level charge transfer. Following this charge transfer, the final time dependent electron wavefunctions are linear combinations of a number of eigenfunctions. Each such eigen state has different electron occupations. In Fig. 4, we show some possible states with different electron occupations. To simplify, we neglect electron spins and only depict the intra-gap levels and their occupations. State (a) is the initial electron configuration which is identical to the configuration shown in Fig. 1(b). State (b) is obtained from state (a) by transferring one electron from $\varepsilon_{\mathrm{BP}^{-}}^{\mathrm{u}}$ to $\varepsilon_{\mathrm{BP}^{+}}^{\mathrm{u}}$ and to get state (c) one electron is transferred from $\varepsilon_{\mathrm{BP}^{-}}^{\mathrm{d}}$ to $\varepsilon_{\mathrm{BP}^{+}}^{\mathrm{d}}$. Considering the electron spin, states (b) and (c) correspond two electron configurations, respectively.

In states (d)-(g), there are only two intra-gap levels $\varepsilon_{1}^{\mathrm{u}}$ and $\varepsilon_{1}^{\mathrm{d}}$, which describe the intra-gap levels shown for $t>900$ fs in Fig. 3, panels a1 and b1. To obtain state (d), two electrons are transferred from $\varepsilon_{\mathrm{BP}^{-}}^{\mathrm{u}}$ to $\varepsilon_{\mathrm{BP}^{+}}^{\mathrm{u}}$ and from $\varepsilon_{\mathrm{BP}^{-}}^{\mathrm{d}}$ to $\varepsilon_{\mathrm{BP}^{+}}^{\mathrm{d}}$, respectively. All the levels in conduction band are doubly occupied and all the levels in valence band are empty, consequently state (d) denotes a biexciton state. In state (e), all the levels in conduction band are empty and there is a hole in valence band, thus it denotes an excited negative polaron and a "free" hole. In state (f), in addition to the occupation of $\varepsilon_{1}^{\mathrm{u}}$, all the levels in valence band are doubly occupied and

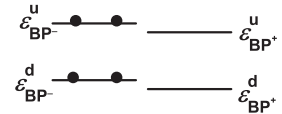

(a) $\mathrm{BP}^{2-}+\mathrm{BP}^{2+}$

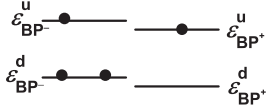

(b) $\mathrm{P}^{-}+\mathrm{P}^{*+}$

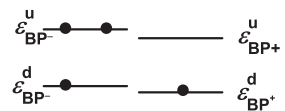

(c) $P^{*}+P^{+}$

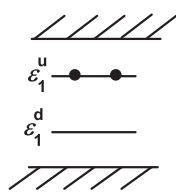

(d) $\mathrm{BX}$

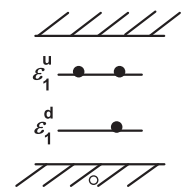

(e) $\mathrm{P}^{*-}+\mathrm{h}$

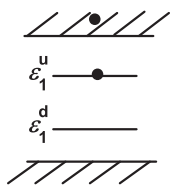

(f) $\mathrm{P}^{*+}+\mathrm{e}$

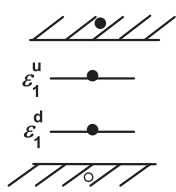

(g) $E x+e+h$
FIG. 4. The possible states with different electron occupations during the bipolaron recombination process. $\mathrm{BP}^{2+}\left(\mathrm{BP}^{2-}\right)$ denote a positive (negative) bipolaron, $\mathrm{BX}$ a biexciton, $\mathrm{P}^{*-}\left(\mathrm{P}^{*+}\right)$ a excited negative (positive) polaron, $\mathrm{h}$ (e) a free hole (electron), and EX an exciton. there is an electron in conduction band, so it denotes an excited positive polaron and a "free" electron. State (g), finally, corresponds to an exciton, and in addition a free electron and a free hole. Apparently, state (e)-(g) correspond to a large number of electron configurations. As discussed below, calculations show that the yields of these states dominate the states after the bipolaron recombination.

In the following, we calculate the yields for different states that are associated with the bipolaron scattering process using a projection method. ${ }^{28,29}$ The evolved wavefunction $|\Psi(t)\rangle$ can be constructed by the evolved single electron wavefunctions $\left\{\psi_{k}(n, t)\right\}$ as a Slater determinant. The eigenfunction $\left|\Phi_{K}\right\rangle$ corresponding to a electron configuration is also constructed as a Slater determinant by the single electron eigenfunctions of the Hamiltonian $H_{\text {elec }}$, i.e., $\left\{\phi_{k}(n, t)\right\}$. After each evolution step, the evolved wavefunction $|\Psi(t)\rangle$ is projected onto the eigenfunction $\left|\Phi_{K}\right\rangle$. The relative yield $I_{K}(t)$ for this electron configuration is then obtained from

$$
I_{K}(t)=\left|\left\langle\Phi_{K} \mid \Psi(t)\right\rangle\right|^{2} .
$$

$\left|\Phi_{K}\right\rangle$ can be any electron configuration of interest. In calculating the yields of state (b), (c), and (e)-(g) in Fig. 4, we add the yields of all possible configurations.

In Fig. 5, we show time dependence of the yields for the states displayed in Fig. 4. Before the two bipolarons collision, we see that the yield of state (a) is $100 \%$. After the first collision at about $500 \mathrm{fs}$, the yield of state (a) decreases from $100 \%$ to about $85 \%$, while at the same time the yields of states (b) and (c) increase from zero to about 5\% and 10\%, respectively. This change is associated with a small amount of charge transfer from $\varepsilon_{1}^{\mathrm{u}}$ to $\varepsilon_{2}^{\mathrm{u}}$ and from $\varepsilon_{1}^{\mathrm{d}}$ to $\varepsilon_{2}^{\mathrm{d}}$ (i.e., from chain 1 to chain 2). After the second collision at about $900 \mathrm{fs}$, we see that the yields of states (a)-(c) sharply reduce to zero. At the same time, after some oscillations, the yield of

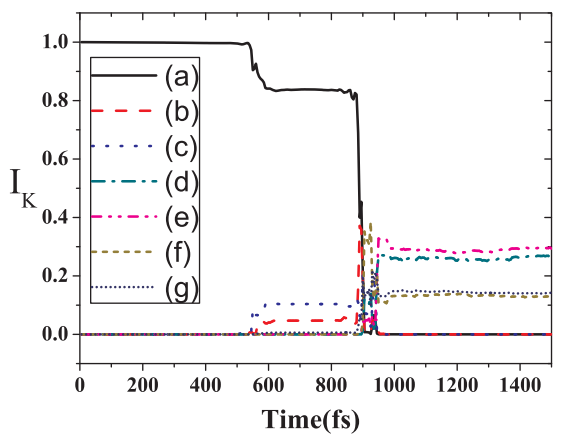

FIG. 5. Time dependence of the yields of states (a)-(g) which are shown in Fig. $4, U_{0}=0.3 t_{0}$. 
states (d)-(g) increase from zero to about 26\%, 29\%, $13 \%$, and $14 \%$, respectively.

The results above indicate the following bipolaron recombination reaction channels (see notations in Fig. 4):

$$
\begin{gathered}
\mathrm{BP}^{2+}+\mathrm{BP}^{2-} \rightarrow \mathrm{BX}, \\
\mathrm{BP}^{2+}+\mathrm{BP}^{2-} \rightarrow \mathrm{P}^{*-}+h, \\
\mathrm{BP}^{2+}+\mathrm{BP}^{2-} \rightarrow \mathrm{P}^{*+}+e, \\
\mathrm{BP}^{2+}+\mathrm{BP}^{2-} \rightarrow \mathrm{EX}+e+h .
\end{gathered}
$$

These reaction channels clearly show that other localized excited states, beside the biexciton state, can be produced with certain yields during the bipolaron recombination. This is reasonable because of the total energy conservation: from Fig. 4, it is easy to infer that the energy of the localized excited states (e)-(g) are all higher than that of state (d) (the biexciton state). This guarantees the total energy conservation during the bipolaron recombination.

In addition to the four channels listed above, there are a large number of other states involved in the recombination. Together they contribute the remaining $18 \%$ of the total yield. However, each individual such state has very small yield and is therefore of less interest as concerns the recombination process.

As a final remark it should be noted that the individual states have their own time dynamics following the recombination. However, in the way the time evolution is treated here, we obtain a weighted average of the time dependence and cannot follow the states individually. This is the reason why, for instance, the (hot) electron-hole pair described in Eq. (22) above does not decay into an exciton.

\section{CONCLUSIONS}

We have simulated the scattering process between a negative and a positive bipolaron in a system which is composed of two coupled polymer chains. The simulations are performed using a nonadiabatic evolution method, in which the electron wave function is described by the time-dependent Schrödinger equation while the polymer lattice is treated classically by a Newtonian equation of motion.

First we studied the influence of the Coulomb interactions on this scattering process. It is found that if the onsite Coulomb interaction $U_{0}$ is lower than $0.4 t_{0}(1.0 \mathrm{eV})$, the two bipolarons can recombine into a localized excited state. However, if the on-site Coulomb interaction $U_{0}$ is equal to or greater than $0.4 t_{0}$, the two bipolarons never recombine. By a projection method, we found that there are mainly four channels for the bipolaron recombination reaction: (1) forming a biexciton, (2) forming an excited negative polaron and a free hole, (3) forming an excited positive polaron and a free electron and (4) forming an exciton, a free electron and a free hole. In the case of $U_{0}=0.3 t_{0}(0.75 \mathrm{eV})$, the yields for the four channels are $26 \%, 29 \%, 13 \%$, and 14\%, respectively. These numbers provide an insight into how bipolaron recombination occurs. Since there is a considerably higher energy involved in this process as compared to polaron recombination, there are also a larger number of final states accessible. These states will have their own dynamics, which involves both dipole allowed and non-radiative transitions. Studies of these processes are, however, left for coming work.

\section{ACKNOWLEDGMENTS}

Financial support from the Swedish Research Council and from the Swedish Energy Agency is gratefully acknowledged.

${ }^{1}$ A. J. Heeger, S. Kivelson, J. R. Schrieffer, and W. P. Su, Mod. Phys. 60, 781 (1998)

${ }^{2}$ F. Genoud, M. Guglielmi, M. Nechtschein, E. Genies, and M. Salmon, Phys. Rev. Lett. 55, 118 (1985).

${ }^{3}$ Y. Furukawa, J. Phys. Chem. 100, 15644 (1996).

${ }^{4}$ Y. Shimoi and S. Abe, Phys. Rev. B 50, 14781 (1994).

${ }^{5}$ C. H. Lee, G. Yu, and A. J. Heeger, Phys. Rev. B 47, 15543 (1993).

${ }^{6}$ Y. Shimoi and S. Abe, Phys. Rev. B 50, 14781 (1994).

${ }^{7}$ M. N. Bussac and L. Zuppiroli, Phys. Rev. B 49, 5876 (1994).

${ }^{8}$ P. S. Davids, A. Saxena, and D. L. Smith, Phys. Rev. B 53, 4823 (1996).

${ }^{9}$ M. N. Bussac, D. Michoud, and L. Zuppiroli, Phys. Rev. Lett. 81, 1678 (1998)

${ }^{10}$ S. Brazovskii, N. Kirova, Z. G. Yu, A. R. Bishop, and A. Saxena, Synth. Met. 101, 325 (1999).

${ }^{11}$ J. Cornil, D. Beljonne, and J. L. Brédas, J. Chem. Phys. 103, 842 (1995).

${ }^{12} \mathrm{P}$. A. Lane, X. Wei, and Z. V. Vardeny, in Spin-dependent Recombination Processes in $\pi$-Conjugated Polymers, edited by N. S. Sariciftci (World Scientific, Singapore, 1997), p. 292.

${ }^{13}$ P. A. Lane, X. Wei, and Z. V. Vardeny, Phys. Rev. Lett. 77, 1544 (1996).

${ }^{14}$ P. A. Bobbert, T. D. Nguyen, F. W. A. van Oost, B. Koopmans, and M. Wohlgenannt, Phys. Rev. Lett. 99, 216801 (2007).

${ }^{15}$ J. H. Burroughes, D. D. C. Bradley, A. R. Brown, R. N. Marks, K. Mackay, R. H. Friend, P. L. Burn, and A. B. Holmes, Nature (London) 347, 539 (1990).

${ }^{16}$ M. A. Baldo, D. F. O’Brien, M. E. Thompson, and S. R. Forrest, Phys. Rev. B 60, 14422 (1999).

${ }^{17}$ Y. Cao, I. D. Parker, G. Yu, C. Zhang, and A. J. Heeger, Nature (London) 397, 414 (1999)

${ }^{18}$ P. K. H. Ho, J. Kim, J. H. Burroughes, H. Becker, S. F. Y. Li, T. M. Brown, F. Cacialli, and R. H. Friend, Nature (London) 404, 481 (2000).

${ }^{19}$ M. Wohlgenannt, K. Tandon, S. Mazumdar, S. Ramasessha, and Z. V. Vardeny, Nature (London) 409, 494 (2001).

${ }^{20}$ J. S. Wilson, A. S. Dhoot, A. J. A. B. Seeley, M. S. Khan, A. Köhler, and R. H. Friend, Nature (London) 413, 828 (2001).

${ }^{21}$ V. A. Shakin and S. Abe, Phys. Rev. B 50, 4306 (1994).

${ }^{22}$ F. Guo, M. Chandross, and S. Mazumdar, Phys. Rev. Lett. 74, 2086 (1995).

${ }^{23}$ M. A. Pasquinelli and D. Yaron, J. Chem. Phys. 118, 8082 (2003).

${ }^{24}$ V. I. Klimov, D. W. McBranch, N. Barashkov, and J. Ferraris, Phys. Rev. B 58, 7654 (1998)

${ }^{25}$ W. P. Su, J. R. Schrieffer, and A. J. Heeger, Phys. Rev. Lett. 42, 1698 (1979).

${ }^{26}$ Y. Ono and A. Terai, J. Phys. Soc. Jpn. 59, 2893 (1990).

${ }^{27}$ Z. Sun, D. Liu, S. Stafström, and Z. An, J. Chem. Phys. 134, 044906 (2011).

${ }^{28}$ Z. Sun, Y. Li, K. Gao, D. S. Liu, Z. An, and S. J. Xie, Org. Electron. 11, 279 (2010)

${ }^{29}$ Z. Sun, Y. Li, S. J. Xie, Z. An, and D. S. Liu, Phys. Rev. B 79, 201310(R) (2009)

$30 \AA$. Johansson and S. Stafström, Phys. Rev. B 69, 235205 (2004). 